\title{
reverse engineering
}

\section{How we made the liquid crystal display}

\author{
The discovery of the twisted nematic effect allowed liquid crystal displays to become a practical and ubiquitous \\ technology. Martin Schadt recounts how it came about.
}

\section{Martin Schadt}

\begin{abstract}
n 1962, Richard Williams, working at the RCA Laboratories in Princeton, New Jersey, filed the first liquid crystal display
\end{abstract} (LCD) patent, after rediscovering currentinduced light scattering in negative dielectric anisotropic liquid crystal films. (The effect had, in fact, also been observed by Viktor Nikolaevich Zwetkoff in the 1930s.) George Heilmeier and Louis Zanoni continued this work at the RCA Laboratories and in 1968 introduced patterned transparent electrodes and created dynamic scattering LCDs.

These displays suffered from a number of drawbacks including high electrical current consumption, but were, nevertheless, used in early digital watches and pocket calculators.

Inspired by the work at the RCA

Laboratories, the pharmaceutical company Roche, based in Basel, Switzerland, created a liquid crystal research group as part of their strategy to diversify into medical electronics. Wolfgang Helfrich and I were among the first physicists to be hired. Our goal was to find an electro-optical effect that did not rely on current flow, like dynamic scattering LCDs, and instead used an electric field to realign ordered liquid crystal molecules - an approach that would require very little power.

In the fall of 1970, we started investigating the electro-optical properties of twisted configurations of positive nematic liquid crystals, in which the long axes of the molecules tend to point in a direction parallel to an electric field. I initially used $p$-ethoxybenzylidene- $p^{\prime}$-aminobenzonitrile or PEBAB, that my colleague Hanspeter Scherrer had synthesized, even though its melting temperature $\left(106^{\circ} \mathrm{C}\right)$ was impractically high. A film of the molecules was placed between two transparent electrodes, and this cell was placed between two polarizers, which were rotated $90^{\circ}$ relative to each other. The hope was that, in the absence of an electric field (the 'off' state), the arrangement of the twisted liquid crystals would rotate incoming linearly polarized light achromatically by $90^{\circ}$, allowing it to pass through the second polarizer without any dynamic scattering. When an electric field was applied across the electrodes (the 'on' state), the PEBAB molecules would align with the external field and would no longer rotate the incoming polarized light. As a result, the light would be blocked by the second polarizer.

After extensive tests, the experiments were a success and showed that even small voltages could sufficiently deform and unwind the twisted liquid crystal configuration so as to switch a display pixel from transparent to black. The effect became known as the twisted nematic effect, and we filed for a patent on it in December 1970 and published the results in Applied Physics Letters in February 1971.

However, the potential of twisted nematic LCDs (Fig. 1) remained uncertain at this time. It was, in particular, unclear whether stable alignments and reliable room-temperature nematic liquid crystals could be found. The fact that the approach required polarizers, which lead to a reduction in brightness, was also potentially problematic. At Roche, doubts about the technology emerged, and the liquid crystal research project was temporarily stopped. As a result, Helfrich left for the Freie Universität Berlin. I was also about to leave Roche, but in the end stayed, and for the next two years investigated electro-optical effects and charge-transport phenomena of drugs in artificial cell membranes.

In 1973, the Japanese company Seiko approached Roche and offered to buy the rights to the twisted nematic patent. I suggested to our legal department not to sell the patent but to non-exclusively license it to the emerging LCD industry. Roche followed this advice and resumed its liquid crystal research programme. I was given the opportunity to establish a liquid crystal research team composed of physicists and synthetic chemists. Together we developed a room-temperature twisted nematic liquid crystal mixture that was manufactured by Roche and helped the company become one of the leading suppliers of liquid crystals.

A feature key to the function of LCDs is the ability of the molecules to align at the boundaries of displays. This was, at first, achieved with mechanically brushed polymer films. I had long been puzzled by what molecular interactions created this alignment and whether it could be achieved optically. This led in 1992 to the development of a technology to create stable liquid crystal alignment and patterning using polymers

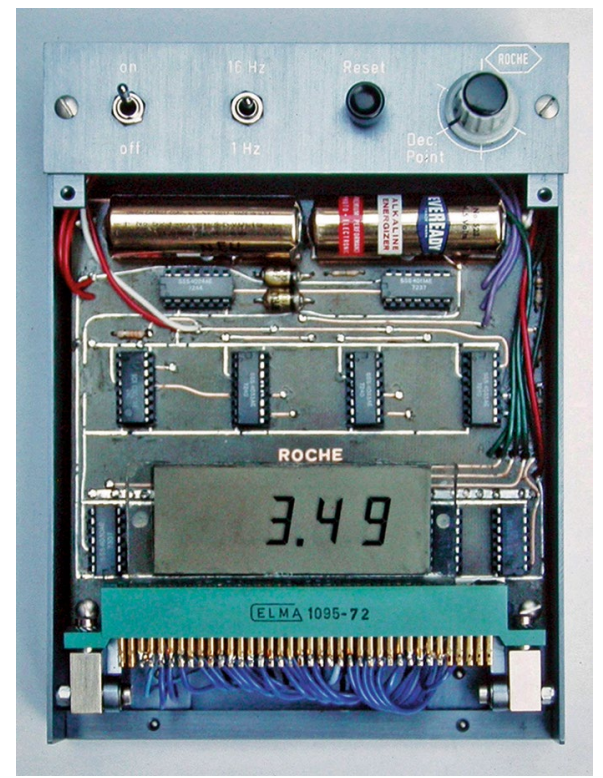

Fig. 1 | A twisted nematic LCD prototype made by the author in 1972.

that can be polymerized in a particular direction by linearly polarized UV light.

Twisted nematic LCDs require low driving voltages and consume virtually zero power. Their stacked electro-optical components are individually tunable prior to integration into the display. This building block concept has allowed technological progress to be incorporated without the redesign of the basic liquid crystal field-effect operating principles. As such, twisted nematic LCDs became the dominant display technology for the rapid expansion of portable electronics during the 1980s and beyond. More recently, different field-effect liquid crystal display technologies have emerged, including in-plane switching and vertical alignment, but the influence of twisted nematic LCDs is still felt today across the ubiquitous use of LCDs from portable devices to high-definition televisions.

\section{Martin Schadt}

Seltisberg, Switzerland.

e-mail:martin.schadt@bluewin.ch

Published online: 13 August 2018

https://doi.org/10.1038/s41928-018-0119-8 